\title{
5. IN CONCLUSION
}

If I sound critical of the book, it is because I basically like it, and I am somewhat disappointed with the final result. Most of the faults could have been easily eliminated by an expert in lattice theory in a few days. And why are words misspelled in these days of spelling checkers? Why is a section heading abbreviated to make it mathematically meaningless ( $\S 5$ of Chapter Two)?

More important than these faults is the enthusiasm of the author for the subject matter. Everybody I discussed this book with became quite enchanted with UC lattices. This topic is full of surprising and deep results, yet even some of the most fundamental problems are unresolved. To whet the reader's appetite, let me mention three:

1. Is there a complete nondistributive UC lattice?

2. Is the MacNeille completion of a UC lattice a UC lattice?

3. Is there a simple "natural" example of a nondistributive UC lattice (in geometry, combinatorics, or topology)?

The first two problems are due to R. P. Dilworth; note that the MacNeille completion of a distributive lattice is not necessarily a distributive lattice. The first explicit mention of the third problem seems to be in the reviewer's General Lattice Theory, but I am sure that experts in the field considered it earlier.

G. GRÄTZER

UNIVERSITY OF MANITOBA

BULLETIN (New Series) OF THE

AMERICAN MATHEMATICAL SOCIETY

Volume 22, Number 2, April 1990

(C) 1990 American Mathematical Society

$0273-0979 / 90 \$ 1.00+\$ .25$ per page

Grassmannians and Gauss maps in piecewise-linear topology, by Norman Levitt. Lecture Notes in Mathematics, vol., SpringerVerlag, 1989, \$21.10. ISBN 0-50756-6

Combinatorial geometry goes back at least to Euclid and the study of regular polyhedrons in Euclidean space, using both metric and linear properties. In the 18th and 19th centuries, Euler and Poincare exploited purely combinatorial properties to define the Euler class, homology and Poincare duality of general polyhedral surfaces; and Riemann exploited local metric properties of smooth 
surfaces to define geodesics and curvature. With the development of topology in the first half of this century, it was shown that the Euler class, homology and Poincaré duality were independent of both the metric and combinatorial structure and were invariants of "bending and stretching." Also, the Chern-Weil theorem showed that real characteristic classes, including the Euler class of smooth manifolds could be defined by differential forms.

Much of the development of geometric topology in the last thirty years has been an effort to study properties of smooth and topological manifolds which were independent of both linear and metric assumptions. Topological and smooth manifolds are simpler to define than combinatorial manifolds, and many of the tools needed, such as bundle theory and automorphism groups are especially difficult in the PL (piecewise linear) category. (The group of PL automorphisms of Euclidean space has no natural topology and is best understood as a simplicial set.) PL topology was important in the development of geometric topology, in particular the $s$-cobordism theorem, and formed a bridge between topological and smooth manifolds for smoothing theory; but was pushed aside when results had been established in the topological category.

On the other hand, the continued interest in Riemannian geometry and local properties such as curvature in both mathematics and physics suggests that local properties of combinatorial manifolds would be of considerable interest. After all the Euler class and the Stiefel-Whitney classes have exceptionally simple definitions in terms of the local combinatorial structure. (The dual homology class of the $i$ th Stiefel Whitney class is represented by the sum of all $n-i$ dimensional simplices of the first barycentric subdivision.) The book under review begins with Levitt and Rourke's proof of the existence of local combinatorial formulae for arbitrary rational characteristic classes. Unfortunately this existence theorem does not exhibit an explicit formula for such classes. The technique is never the less interesting in that it uses a combinatorial version of the Grassman manifold of $n$ planes in infinite dimensional Euclidean space and an explicit "Gauss map" for the normal block bundle of an $n$-dimensional PL manifold $M$ embedded as the diagonal in $M \times M$.

The main thrust of the book is the development of various versions of the Grassmannians of $n$-planes in $n+k$ space which are suitable for triangulated homology manifolds, piecewise smooth embeddings and equivariant immersions and smoothing theory. 
These refine and strengthen the now classical versions of PL immersion theory, equivariant immersion theory and smoothing theory. This extra information enables one to exploit local geometric properties such as D. Stone's notion of curvature of PL immersions. The construction of the PL Grassmannian and associated universal PL bundle is more complicated than in the smooth category, but it is quite natural. It abstracts the notion of link in a combinatorial manifold and has one $j$-cell for each $j$-dimensional abstract link. The book is written for experts and assumes a thorough knowledge of PL topology, bundles and smoothing theory.

Levitt has successfully reintroduced local geometry into the PL category. It remains to see if sufficiently simple local formulas for characteristic classes or sufficiently interesting global results involving curvature etc., can be obtained to justify the conceptual complications introduced by using the PL category.

R. LASHOF

UNIVERSITY OF CHICAGO

BULLETIN (New Series) OF THE

AMERICAN MATHEMATICAL SOCIETY

Volume 22, Number 2, April 1990

(C) 1990 American Mathematical Society

$0273-0979 / 90 \$ 1.00+\$ .25$ per page

Predicative arithmetic, by Edward Nelson. Mathematical Notes, vol. 32, Princeton University Press, Princeton, 1986, vii+189 pp., $\$ 21.00$ (paperback). ISBN 0-691-08455-6

This book presents a formalist account of the foundations of arithmetic and "to one who takes a formalist view of mathematics", Nelson reminds us in his penultimate chapter, "the subject matter of mathematics is the expressions themselves together with the rules for manipulating them-nothing more." This view is expressed even more forcefully in the final sentence of the book: "I hope that mathematics shorn of semantical content will prove useful as we expore new terrain." Now these views are not, of course, new or even particularly extreme but the reader who has reached this point in the book will have realised just how much of conventional mathematical reasoning, and even reasoning usually accepted as totally finitary, Nelson regards as containing unjustifiable semantic elements. Let me, therefore, now turn to the beginning of the book and present some examples of arguments that Nelson finds problematic. 